\title{
Saw Palmetto Therapy for Lower Urinary Tract Symptoms Associated with Benign Prostatic Hyperplasia Assessment in Iraq
}

\author{
Mazin Abdulridha Ateyah ${ }^{1}$, Manal Khalid Abdulridha², Munaim Jumaa Alkabee ${ }^{3}$ \\ ${ }^{1}$ Pharmacist, Clinical Pharmacist, Baghdad, Iraq, ${ }^{2}$ Assist. Prof., Department of Clinical Pharmacy/College of \\ Pharmacy/Mustansiriyah University, Iraq, ${ }^{3}$ Consultant Urosurgeon, Alshahed Alsader Hospital-MOH, Iraq
}

\begin{abstract}
Background: The current study was designed to explore the effectiveness of Saw Palmetto as monotherapy or supplementation with tamsulosin in the treatment of patients with lower urinary tract symptoms consistent with benign prostatic hyperplasia.
\end{abstract}

Method: The present study is diagnosed patient with moderate to severe symptomatic BPH. (60) Patients were divided into 3 groups; Group (1) treated with Saw Palmetto cap (320 mg); Group (2) treated with Saw Palmetto cap $(320 \mathrm{mg})$ and tamsulosin $(0.4 \mathrm{mg})$; Group (3) treated with tamsulosin $(0.4 \mathrm{mg})$, daily for 3 months. The assessment is done based on the change in urological IPSS, urodynamic activity, urine flow, voiding quantity measures, and prostate volume. Also, some measurement such as erection function, urinary incontinence, sleeps quality, and quality of life.

Results: Saw Palmetto supplementation with tamsulosin revealed a significant decrease in the IPSS score $(P<0.01)$, a significant increase in the urine flow rate and the voided volume, a significant decrease in the post-void residual volume and prostate volume $(P<0.01)$, and decrease in serum PSA level $(P<0.01)$. Additionally, improved erection function decreased urinary incontinence score, improvement in sleep quality, and improvement in the quality of life score among the Saw Palmetto supplementation group was noticed $(P<0.01)$.

Conclusion: The phytotherapy with saw palmetto alone or as supplement collectively produced marked improvement in objective and subjective measurements in men with lower urinary tract symptoms consistent with BPH:

Keywords: Benign prostatic hyperplasia, LUTS, Saw palmetto.

\section{Introduction}

Lower urinary tract symptoms LUTS are prevalent in adult men and are often associated with the presence of $\mathrm{BPH}$, which is a troublesome condition that can have a significant negative impact on patients' QoL [1]. The extraction of the Saw Palmetto plant is used most commonly for the treatment of many diseases. In Germany and France, the plant extracts have more than $50 \%$ of all drugs used for BPH therapy. These agents are common used in the United States. Both Boyle in 2004 and Maccagnano in 2006 claimed the efficacy of Permixon ${ }^{\circledR}$ by its comparative effectiveness to $\alpha$ blockers and $5 \alpha$-reductase inhibitors ${ }^{[2]}$.
Still controversy in the therapeutic benefit of combining Saw Palmetto plant extract with the conventional therapy of BPH. The review included 32 randomized controlled trials involving 5666 men, compared with placebo, Serenoa repens at double and triple the usual dose, provides no improvement for nocturia, Qmax, and symptom scores for men with benign prostatic hyperplasia ${ }^{[3]}$. Inversely, another systematic review of 18 randomized controlled trials involving 2939 men with BPH showed significant improvement in urinary tract symptoms and peak urine flow following supplementation with Saw Palmetto plantextract. The update of Cochrane Complementary Medicine stated that Serenoa repens provides mild 
to moderate improvements in urinary symptoms and urinary flowrate ${ }^{[4]}$.

Consumption of herbal drug products by Iraqi patients is quite high. Considering the magnitude of the popularity of these products among the consumers, it is necessary to evaluate the safety, efficacy, and quality of these products which may involve clinical trial studies ${ }^{[5]}$. To the best knowledge, no available data highlight the substantial benefit of Saw Palmetto supplementation as monotherapy or as an adjuvant to other medication therapy, besides the lack of awareness by most urologist about the impact of dietary supplements to provide some evidence-based findings.

\section{Patients and Method}

Patients: The study is used data of patient who attended the private clinic neurosurgeon diagnosed with benign prostatic hyperplasia. Out of 155 patients, only 60 patients completed the study intervention all $\geq 45$ years of age ${ }^{[6]}$.

The patients were divided into three groups; Group (1) included 20 patients treated with Saw palmetto (320 mg Serenoarepenes) to be given once daily for 12 weeks; Group (2) included 20 patients treated with Saw palmetto (320 mg Serenoarepenes) and tamsulosine (0.4) $\mathrm{mg}$ to be given once daily for 12 weeks; Group (3) included 20 patients treated with tamsulosin $(0.4) \mathrm{mg}$ to be given once daily for 3 months.

\section{Method}

American Urological Association Symptom Score questionnaire have seven questions intended to classify the severity of enlarged prostate symptoms that were carried out at baseline, after 4 weeks, after 8 weeks, and after 12 weeks ${ }^{[13]}$.All other objective measurements were (voided volume, post-void residual volume, prostate volume, and prostate-specific antigene) carried out at baseline and after 12 weeks.

All subjective questionnaires were carried out at baseline, after 1 month, after 2 months, and after 3 months. Erection function assessed using International Index of Erectile Functions (ILEF) questions (1, 2, 3, 4,5 , and 15) Patients with ILEF scores $(<14$ out of 30$)$ in Domain A (Erectile Function) considered low. The Urinary Incontinence assessed by 0-24 incontinence symptoms subscale (IS) from International Consultation on Incontinence Questionnaire Male Lower Urinary Tract Symptoms. The four-item Jenkins Sleep scale was used to assess and evaluate the urinary function for patients at different levels. The questionnaire evaluated the frequency and intensity of certain sleep difficulties in respondents the value of the Jenkins Sleep scale Questionnaire is $(0-20)^{[16]}$.

\section{Results}

Patient demographic and disease characteristics: The patient demographic of a total of 60 patients included in the present study is shown in table (1).There was no statistically significant difference between study groups concerning age, BMI, and duration of symptoms, $(P \geq 0.05)$.

Table (1): Disease characteristics with patients demographic

\begin{tabular}{|c|c|c|c|c|c|c|c|c|}
\hline \multicolumn{9}{|c|}{ Study Groups } \\
\hline \multirow{2}{*}{\multicolumn{2}{|c|}{ Study variable }} & \multicolumn{2}{|c|}{ Group 1} & \multicolumn{2}{|c|}{ Group 2} & \multicolumn{2}{|c|}{ Group 3} & \multirow{2}{*}{ P-value } \\
\hline & & $\mathbf{n}$ & $\%$ & $\mathbf{n}$ & $\%$ & $\mathbf{n}$ & $\%$ & \\
\hline \multirow{3}{*}{ Age (Year) } & $<55$ & 3 & $25.0 \%$ & 6 & $50.0 \%$ & 3 & $25.0 \%$ & \multirow{3}{*}{$0.481^{\mathrm{NS}}$} \\
\hline & $55-64$ & 8 & $47.1 \%$ & 4 & $23.5 \%$ & 5 & $29.4 \%$ & \\
\hline & $64>$ & 9 & $29.0 \%$ & 10 & $32.3 \%$ & 12 & $38.7 \%$ & \\
\hline \multicolumn{2}{|c|}{ BMI $\left(\mathrm{kg} / \mathrm{m}^{2}\right)$} & \multicolumn{2}{|c|}{$27 \pm 2.7$} & \multicolumn{2}{|c|}{$27.4 \pm 2.6$} & \multicolumn{2}{|c|}{$25.8 \pm 2.5$} & $0.142^{\mathrm{NS}}$ \\
\hline \multicolumn{2}{|c|}{ Duration of symptoms (Months) } & \multicolumn{2}{|c|}{$9.4 \pm 2.4$} & \multicolumn{2}{|c|}{$7.1 \pm 1.4$} & \multicolumn{2}{|c|}{$7.7 \pm 2.2$} & $0.107^{\mathrm{NS}}$ \\
\hline
\end{tabular}

Data expressed as mean \pm SD, Percentage (\%), Number of patients (n), Chi-square is used for statistical analysis. NS: No significant differences $(\mathrm{P} \geq 0.05)$. 
Effect of study intervention on objective parameters: There are no significant differences $(\mathrm{P} \geq 0.05)$ in the urine flow rate between study groups pre and post-treatment, Table (2). Within each study group, there was a highly significant increase $(\mathrm{P}<0.01)$ in the urine flow rate at the end of the study, with a high percentage of change, noticed among group 2 patients $(52.25 \%)$. Significant differences $(\mathrm{P}<0.01)$ in voided volume were revealed between the study group posttreatment only. Also, there was a highly significant increase $(\mathrm{P}<0.01)$ in the voided volume after three months, mainly among group 3 patients $(82.61 \%)$. The present study showed highly significant differences $(\mathrm{P}<0.01)$ in post-void residual volume between study groups post-treatment only, and the significant decrease
$(\mathrm{P}<0.01)$ in the post-void residual volume was noticed among group 2 patients $(59.36 \%)$ after three months of treatment. Moreover, results showed no significant differences $(\mathrm{P} \geq 0.05)$ in prostate volume between study groups pre and post-treatment. Nevertheless, there was a highly significant decrease $(\mathrm{P}<0.01)$ within each study group in the prostate volume after three months, particularly, among group 2 patients high percent of change was noticed(3.38\%). The changes in serum PSA level revealed a significant difference between groups 1and 2 at the end of treatment $(\mathrm{P}<0.05)$. Patients in group 2 presented with a high percentage of change in the serum PSA level (-10.59\%) compared to other study groups after treatment $(\mathrm{P}<0.01)$.

Table (2): Effect of study intervention on objective parameters pre and post-treatment

\begin{tabular}{|c|c|c|c|c|}
\hline \multirow{2}{*}{ Study ariables } & \multicolumn{3}{|c|}{ Study groups } & \multirow{2}{*}{ Pb value } \\
\hline & Group 1 & Group 2 & Group 3 & \\
\hline \multicolumn{5}{|c|}{ Urine flow rate $(\mathrm{ml} / \mathrm{s})$} \\
\hline Pre- treatment & $8.18+2.21$ & $7.98+2.32$ & $7.40+1.90$ & $0.50^{\mathrm{NS}}$ \\
\hline Post- treatment & $11.58+3.68$ & $12.15+4.00$ & $10.79+3.75$ & $0.53^{\mathrm{NS}}$ \\
\hline $\mathrm{Pa}$ value & $0.001 * *$ & $0.001 * *$ & $0.001^{* *}$ & \\
\hline Change (\%) & $41.56 \%$ & $52.25 \%$ & $45.81 \%$ & \\
\hline \multicolumn{5}{|c|}{ Voided Volume (ml) } \\
\hline Pre-treatment & $152.75+15.93$ & $164.00+37.16$ & $151.00+14.29$ & $0.206^{\mathrm{NS}}$ \\
\hline Post-treatment & $249.75+42.93$ & $295.50+31.07$ & $275.75+40.56$ & $0.002 * *$ \\
\hline Pa value & $0.001 * *$ & $0.001 * *$ & $0.001 * *$ & \\
\hline Change $(\%)$ & $63.50 \%$ & $80.18 \%$ & $82.61 \%$ & \\
\hline \multicolumn{5}{|c|}{ Post Void Residual Volume (ml) } \\
\hline Pre- treatment & $216.25+39.23$ & $220.25+48.00$ & $219.50+40.23$ & $0.95^{\mathrm{NS}}$ \\
\hline Post- treatment & $120.25+40.44$ & $89.50+26.45$ & $109.25+36.75$ & $0.02 *$ \\
\hline Pa value & $0.001 * *$ & $0.001 * *$ & $0.001 * *$ & \\
\hline Change (\%) & $-44.39 \%$ & $-59.36 \%$ & $-50.22 \%$ & \\
\hline \multicolumn{5}{|c|}{ Prostate Volume $\left(\mathrm{cm}^{3}\right)$} \\
\hline Pre- treatment & $46.10+6.71$ & $44.35+5.60$ & $46.70+6.62$ & $0.48^{\mathrm{NS}}$ \\
\hline Post- treatment & $45.45+6.95$ & $42.85+4.96$ & $45.70+6.36$ & $0.27^{\mathrm{NS}}$ \\
\hline Pa value & $0.004 * *$ & $0.001 * *$ & $0.001 * *$ & \\
\hline Change (\%) & $-1.40 \%$ & $-3.38 \%$ & $-2.14 \%$ & \\
\hline \multicolumn{5}{|c|}{ PSA (ng/ml) } \\
\hline Pre- treatment & $2.92+2.52$ & $1.51+1.45$ & $1.76+1.51$ & $0.050^{*}$ \\
\hline Post- treatment & $2.83+2.40$ & $1.35+1.31$ & $1.61+1.25$ & $0.019^{*}$ \\
\hline Pa value & $0.001 * *$ & $0.001 * *$ & $0.031 *$ & \\
\hline Change (\%) & $-3.08 \%$ & $-10.59 \%$ & $-8.52 \%$ & \\
\hline
\end{tabular}

Data expressed as mean \pm SD, percentage of change $(\%)$, Number of patients (n),NS: No significant differences $(\mathrm{P} \geq 0.05), * *(\mathrm{P}<0.01)$ is highly significant. (a): Paired t-test is used to compare pre- and post-treatment results in the same group.(b): ANOVA (one way) is used to compare study intervals among group 1 group 2, and group 3 patients 
Effect of study intervention on subjective parameters: There were highly significant differences in the (IPSS/AUA) mean index score among the study groups at baseline, 4 weeks, 8 weeks, and 12 weeks study intervals $(P<0.01)$. Also, the AUA mean score showed a highly significant decrease within each study group at the end of the study $(P<0.01)$, with a high percentage of change noticed with group 2 patients $(-81.68 \%)$, Table (3).No significant differences in the erection function (of the IIEF Questionnaire) mean score was produced among the study groups at baseline, 4, 8; 12 weeks study intervals $(P \geq 0.05)$. Also the erection function means score showed highly significant improvement within each study group at the end of the study $(P<0.01)$, with a high percentage of change noticed among group 2 patients $(108.03 \%)$. There were highly significant differences in the urinary incontinence mean score among the study groups at baseline, 4 weeks, 8 weeks, and 12 weeks study intervals $(P<0.01)$ as in Table (35 ) and Figure (3-4), Also the urinary incontinence mean score showed highly significant decrease within each study group at the end of the study $(P<0.01)$, with a high percentage of change noticed with group 2 patients $(-70.92 \%)$. There were highly significant differences in the quality of sleep mean score among the study groups at baseline, 4 weeks, 8 weeks, and 12 weeks of study intervals $(P<0.01)$.the score quality of sleep showed a highly significant decrease within each study group at the end of the study $(P<0.01)$, with a high percentage of change noticed with group 2 patients $(-82.90 \%)$. There were highly significant differences in the quality of life mean score among the study groups at baseline 4, 8, 12 weeks of study intervals $(P<0.01)$.

Table (3): Effect of study intervention on subjective parameters

\begin{tabular}{|c|c|c|c|c|}
\hline \multirow{2}{*}{ The variables } & \multicolumn{3}{|c|}{ The groups } & \multirow{2}{*}{$P$ value } \\
\hline & Group 1 & Group 2 & Group 3 & \\
\hline \multicolumn{5}{|c|}{ (IPSS) score } \\
\hline Baseline & $21.0+2.88$ & $20.2+3.4$ & $20.6+2.1$ & $0.675^{\mathrm{NS}}$ \\
\hline 4 weeks & $17.9+2.39$ & $10.9+3.0$ & $13.1+2.4$ & $0.000 * *$ \\
\hline 8 weeks & $13.1+3.14$ & $6.3+2.7$ & $8.1+1.7$ & $0.001 * *$ \\
\hline 12 weeks & $8.8+3.81$ & $3.7+1.4$ & $5.3+1.2$ & $0.001 * *$ \\
\hline$P$ value & $0.001 * *$ & $0.001 * *$ & $0.001 * *$ & \\
\hline Change (\%) & $-58.09 \%$ & $-81.68 \%$ & $-74.27 \%$ & \\
\hline \multicolumn{5}{|c|}{ (ILEF) score } \\
\hline Baseline & $7.05+2.67$ & $5.60+2.60$ & $5.65+3.27$ & $0.200^{\mathrm{NS}}$ \\
\hline 4 weeks & $8.30+3.63$ & $7.40+3.62$ & $5.95+3.38$ & $0.116^{\mathrm{NS}}$ \\
\hline 8 weeks & $10.15+4.60$ & $9.85+4.58$ & $7.75+4.12$ & $0.185^{\mathrm{NS}}$ \\
\hline 12 week & $11.55+5.53$ & $11.65+5.61$ & $8.75+4.81$ & $0.158^{\mathrm{NS}}$ \\
\hline $\mathrm{Pa}$ value & $0.001 * *$ & $0.001 * *$ & $0.001 * *$ & \\
\hline Change (\%) & $63.82 \%$ & $108.03 \%$ & $54.86 \%$ & \\
\hline \multicolumn{5}{|c|}{ (UIQ) score } \\
\hline Baseline & $10.90+1.86$ & $11.35+2.08$ & $11.40+1.79$ & $0.63 \mathrm{NS}$ \\
\hline 4 weeks & $9.15+1.79$ & $6.15+1.81$ & $8.10+1.07$ & $0.001 * *$ \\
\hline 8 weeks & $7.15+1.73$ & $4.45+1.88$ & $6.05+1.05$ & $0.001 * *$ \\
\hline 12 weeks & $5.90+1.59$ & $3.30+1.56$ & $4.45+1.19$ & $0.001 * *$ \\
\hline Pa value & $0.001 * *$ & $0.001 * *$ & $0.001 * *$ & \\
\hline Percentage of change $(\%)$ & $-46.11 \%$ & $-70.92 \%$ & $-60.96 \%$ & \\
\hline
\end{tabular}




\begin{tabular}{|c|c|c|c|c|}
\hline \multirow{2}{*}{ The variables } & \multicolumn{3}{|c|}{ The groups } & \multirow{2}{*}{$P$ value } \\
\hline & Group 1 & Group 2 & Group 3 & \\
\hline \multicolumn{5}{|c|}{ (JENKINS) Sleep score } \\
\hline Baseline & $15.80+2.91$ & $13.75+2.83$ & $15.55+1.28$ & $0.021 *$ \\
\hline 4 weeks & $14.05+2.95$ & $7.15+2.18$ & $9.00+2.08$ & $0.001 * *$ \\
\hline 8 weeks & $10.60+3.53$ & $4.20+1.40$ & $5.65+1.87$ & $0.001 * *$ \\
\hline 12 weeks & $7.40+3.59$ & $2.35+1.04$ & $3.40+1.19$ & $0.001 * *$ \\
\hline$P a$ value & $0.001 * *$ & $0.001 * *$ & $0.001 * *$ & \\
\hline Change (\%) & $-53.16 \%$ & $-82.90 \%$ & $-78.13 \%$ & \\
\hline \multicolumn{5}{|c|}{ (QoL) score } \\
\hline Baseline & $4.90+0.31$ & $4.75+0.44$ & $4.70+0.47$ & $0.290 \mathrm{NS}$ \\
\hline 4 weeks & $4.15+0.59$ & $3.10+0.45$ & $3.35+0.49$ & $0.001 * *$ \\
\hline 8 weeks & $3.70+0.57$ & $2.45+0.76$ & $2.70+0.57$ & $0.001 * *$ \\
\hline 12 weeks & $2.50+0.61$ & $1.40+0.99$ & $1.70+0.47$ & $0.001^{* *}$ \\
\hline $\mathrm{P}$ a- value & $0.001 * *$ & $0.001 * *$ & $0.001 * *$ & \\
\hline Change (\%) & $-48.97 \%$ & $-70.52 \%$ & $-63.82 \%$ & \\
\hline
\end{tabular}

Data expressed as mean \pm SD, percentage of change (\%), Number of patients (n),.NS: No significant differences $(\mathrm{P} \geq 0.05), * *(\mathrm{P}<0.01)$ is highly significant. (a): ANOVA is used to compare among study intervals (4,8, 12 weeks) treatment results in the same group.(b): ANOVA test is used to compare study intervals among group 1 group 2,and group 3 patients.

\section{Discussion}

As mentioned earlier, the AUA 2010 guideline update lowered the age of the Index Patient from age 50 years or older to age 45 years or older ${ }^{[7]}$, which applies to the age group of the current study where half of them less than 65 year old, $(20 \%)$ of patients were less than 54year old, (28.3\%) were 55-65year old, and (51.7\%) were older than $64 \mathrm{y}$ old. Postmortem research has seen an 8 percent, 50 percent, and 80 percent incidence respectively for the fourth, sixth, and ninth decades of life ${ }^{[10]}$

Most of the enrolled patients in the current study were diagnosed with moderate to severe symptomatic $\mathrm{BPH}$ and were mostly overweight. The reports showed that obesity has related to BPH, the recent one in 2019 revealed a marked association between the risk of larger $\mathrm{PV}$ and BMI, and suggested considering Concomitant diseases like Obesity and diabetes may influence the risk of BPH and LUTS in older men ${ }^{[11]}$.

AUA Score, Prostate Volume, Urine Flow Rate, Voided Volume, Post-Void Residual Volume:

The effect of Saw Palmetto alone or as a supplement with Tamsulosin on objective measures for LUTS/BPH was reported by many review articles and worldwide studies, but no data available from the Iraqi population yet to the best search. Controversy data obtained from several previous reviews, data from early review stated that Saw Palmetto extract (Serenoa repens) alone, at double and triple doses, did not significantly decrease nightly urination on the AUA, peak urine flow, or prostate size in men with lower urinary tract symptoms consistent with $\mathrm{BPH}^{[3]}$. A study involving more than one thousand patients (55-80) years old with IPSS $\geq 7$, to compare Saw Palmetto extract with tamsulosin in BPH therapy after at six month,also, Serenoa repens used for the same purpose due to it has the same effect in BPH therapy in terms of IPSS $(p=0.20)$, QoL $(p=0.33)$, Qmax $(p=0.21)$, PVR $(p=0.65)$, and PSA $(p=0.08)$ [12].

In a similar 3 groups treatment comparative study, no difference was found in the sense of a change between baseline and final evaluation in total IPSS score, however, during the treatment period $20(23 \%)$ of the patients managed with tamsulosin and 17 (21\%) with tamsulosin plus Saw palmetto had a drug-treated with related adverse reactions ${ }^{[13]}$. The more recent study reported that the long-term prediction of 7 initial criteria (predictors): IPSS; (Qmax); average urine flow rate; 
urination volume, urination time, residual urine volume, prostate volume, used to assess the treatment outcome with a lipidosterolic extract of Serenoa repens $(320 \mathrm{mg}$. Permixon (capsules) per day for two years in LUTS/ BPH patients aged (52 to 87 years) with symptomatic benign prostatic hypertrophy ${ }^{[14]}$. The prostate size increases with age. The prostate are growth with age with four stages with the second stage (50-90) years and the prostate increase $(0.5-1.2)$ gram yearly [15][16].

Several other studies tested the effect of with Saw Palmetto as a monotherapy, a study extended to 12 months in patients with BPH aged (53.3) years. The results showed a simple reduction of prostate size and volume from baseline $(26.1 \pm 1.7)$ vs $(24.8 \pm 1.7)$ respectively. Others reported prostate size decreased after administration of Saw Palmetto (from 51.1 \pm 20.1 to $43.3 \pm 15.9$ ). Additionally, in younger men aged (35.06 \pm 5.85$)$ years on $320 \mathrm{mg}$ Saw Palmetto as a monotherapy showed $(15 \%)$, decrease in prostate size ${ }^{[16]}$. And that is agreement with our study.

Most of the clinical trials use this cut off value as inclusion criteria ${ }^{[17]}$.Several previous comparative studies reported equipotent effect of Saw Palmetto and tamsulosin that matched the current study findings, one of them recorded that the increasing in Qmax was similar in both over 3-12 months period. Another matched findings to the current study, the efficacy of both the Saw Palmetto and tamsulosin groups from day 0 to 6 th month of treatment produced similar the mean changes Qmax in both, and the decreased prostate volume andthe improvement in PVR was noticed in the Saw Palmetto group the most.Additionally, in his review Giacomo $\mathrm{N}$. reported superiority issue of the combination of Permixon and tamsulosin for relieving LUTS $(p<0.01)$ but not in reducing the number of nocturnal voids and increasing Qmax which was explored more in Permixon alone $^{[37]}$. Inversely, prospective open-label, 12-month study stated no differences in maximal urinary flow rate, PVR, prostate-specific antigen, and prostate volume between a combination of (Saw Palmetto with bovine colostrums) ${ }^{[19]}$.

Erection Function, Urinary Incontinence, Sleep Quality, and Quality of Life: Numerous clinical studies with thousands of men have documented that the benefits of saw palmetto can build over time. Compare this to the common drug finasteride (Proscar) which causes erectile dysfunction for about (5\%) of users, a study found that sexual function was significantly improved during the second year of two years taking saw palmetto. A previous review published in The Journal of Urology analyzed (30) in vivo and in vitro laboratory studies, noted that the frequency of use of Saw Palmetto globally for medical treatment of men's disorders, BPH matches the use of conventional drugs, and the studies confirm that Saw Palmetto has a wide spectrum of activity via numerous mechanisms such as subduing excess testosterone and DHT, and a known anti-inflammatory action. ${ }^{[20]}$ In agreement with previous studies.

An enlarged prostate can cause frustrating and uncomfortable symptoms, urinary difficulties, urinary hesitancy, weak stream, and nocturia are the most commonly reported LUTS, it is estimated that $90 \%$ of men between 45 - 80 years of age suffer some types of LUTS. After 3-month treatment protocols in the current study revealed a marked decrease in urinary incontinence mean score up to $(-70.92 \%)$ downscale $(p<0.01)$, particularly among patients received combination therapy, besides saw palmetto or tamsulosin also produced a significant decrease in urinary function in a significant difference between the groups $(p<0.01)^{[21]}$.

The four-item Jenkins Sleep scale Questionnaire in the current study showed a marked decrease within each study group after 12 weeks $(\mathrm{P}<0.01)$,particular change was noticed among group 2 patients received combination therapy $(-82.90 \%)$. Both Saw Palmetto and tamsulosin also produced significant decrease as well. The reason is perhaps due to the effects of those treatments on the GABA neurotransmitter that acts on increasing the capacity of the urinary bladder which decreases the frequent times of waking up during night sleep $^{[22]}$.

Ethical Clearance: The Research Ethical Committee at scientific research by ethical approval of bothMOH and MOHSER in Iraq.

\section{Conflict of Interest: None}

Funding: Self-funding

\section{References:}

1. Welch G, Weinger K, Barry MJ. Quality-of-life impact of lower urinary tract symptom severity: results from the Health Professionals Follow-up Study. Urology 2002; 59: 245-50.

2. Sharma M, Chadha R, Dhingra N. Phytotherapeutic Agents for Benign Prostatic Hyperplasia: An 
Overview. Mini Rev Med Chem. 2017. 17 (14):1346-1363.

3. Tacklind J, MacDonald R, Rutks I, Stanke JU, Wilt TJ. Serenoarepens for benign prostatic hyperplasia. Cochrane Database of Systematic Reviews 2012, 12. Art. No.:CD001423.

4. Carmen Maccagnano, Andrea Salonia, Alberto Briganti, Claude Schulman, Francesco Montorsi, Patrizio Rigatti, A Critical Analysis of Permixon ${ }^{\mathrm{TM}}$ in the Treatment of Lower Urinary Tract Symptoms Due to Benign Prostatic, Enlargement, (2006).

5-Vary K Mc. and Kaplan S., Role of Complimentary Therapy for Male LUTS, (2013).

6. World Health Organization. Declaration of Helsinki. British Medical Journal. 1996; 313(7070): 1448-1449.

7. AUA Practice Guidelines Committee. AUA guideline on the management of benign prostatic hyperplasia (2010).

8. Kadhim Ali Kadhim, Ashwaq Najemaldeen Abbas, Saad Abdulrahman Hussain,Use of herbal drugs as alternative medicine: Experience of Iraqi patients Spatula DD. 2015; 5(4):1-7.

9. Mustafa Safaa Hussain, Manal Khalid Abdulridha, Mahmood Shaker Khudhair, Study the Effect of Evening Primrose Oil Supplement in Type 2 Diabetes Mellitus -Associated Metabolic Parameters, UK Journal of Pharmaceutical and Biosciences 5(2), 25-31, 2017

10. Sura Abbas Khdair, Manal Khalid Abdulridha, Mostafa Abdal Fatah Shafek, Effect of Curcumin Supplement on Pulmonary Functions, Total and Differential White Blood Cell Count, Serum Level of Leptin and Body Mass Index in a Sampleof Iraqi Patients with Chronic Bronchial Asthma. Al Mustansiriyah Journal of Pharmaceutical Sciences, 2019, 19; 47-58.

11. Maiss Saadi Baqer, Mohammed Mahmood Mohammed, Nizar Abdullateef Jassim. Curcumin as adjuvant therapy to Meloxicam in treatment of patients with knee Osteoarthritis; Evaluation of antioxidant activity, Al Mustansiriyah Journal of Pharmaceutical Sciences, (2020): 20:71- 8112

12. Cai, Yuanshan Cui Shaoxia Yu,Qian Li, Zhongbao Zhoul, and Zhenli Gao, Comparison of Serenoarepens with Tamsulosin in the Treatment of Benign Prostatic Hyperplasia: A Systematic
Review and Meta-Analysis American Journal of Men's Health March-April 2020: 1-11.

13. Aleksandar Argiroviü, Djordje Argiroviü, Does the addition of Serenoarepens to tamsulosin improve its therapeutical efficacy in benign prostatic hyperplasia? 2013; 70(12): 1091-1096.

14. Debruyne, F. et al. 'Comparison of a phytotherapeutic agent (Permixon) with an alphablocker (Tamsulosin) in the treatment of benign prostatic hyperplasia: a 1-year randomized international study.' European urology, 2002: 41(5), 497-506.

15. Santillo, Vincent M.; Lowe, Franklin C. Role of Vitamins, Minerals and Supplements In The Prevention And Management of Prostate Cancer. Int. Braz J Urol., Rio De Janeiro, 2006, 32: 3-14.

16. Chokkalingam AP, Yeboah ED, Demarzo A, Netto $\mathrm{G}, \mathrm{Yu} \mathrm{K}$. Prevalence of BPH and lower urinary tract symptoms in West Africans. Prostate Cancer Prostatic Dis 2012; 15: 170-6.

17. Cai T, Mazzoli S, Bechi A, Addonisio P, MondainiN, Pagliai RC, Bartoletti R. Serenoarepens associated with Urticadioica (Prosta MEV) and curcumin and quercitin (FlogMEV) extracts are able to improve the efficacy of prulifloxacin in bacterial prostatitis patients: results from a prospective randomised study. Int J Antimicrob, 2009, 33(6):549-553.

18. Djavan B, Margreiter M, Dianat SS. An algorithm for medical management in male lower urinary tract symptoms. CurrOpin Urol. 2011;21(1):5-12.

19. Di Maida F.a $\cdot$ Mari A.a $\cdot$ RubinoR.b $\cdot$ MinerviniA.a Carini M.a $\cdot$ Siena G.aA, Prospective, Open-Label Comparison of Tamsulosin plus Serenoarepens and Bovine Colostrum versus Tamsulosin Alone in the Treatment of Benign Prostatic Hyperplasia

20. Buck, A. C. Is there a scientific basis for the therapeutic effects of serenoarepens in benign prostatic hyperplasia? Mechanisms of action. The Journal of Urology (2004): 172: 1792-1799.

21. Suzuki, Mayumi et al. "Pharmacological effects of saw palmetto extract in the lower urinary tract." Actapharmacologica Sinica 2009: 3: 227-81.

22. Helfand BT, McVary KT, Meleth S, Sharp V, Foster H, Naslund M, Williams OD; CAMUS Study Group. the relationship between lower urinary tract symptom severity and sleep disturbance in the CAMUS trial. 2011. 